\title{
Substance Use Supplemental Qualifiers Dataset
}

National Cancer Institute

\section{Source}

National Cancer Institute. Substance Use Supplemental Qualifiers Dataset. NCI

Thesaurus. Code C147252.

A dataset containing supplemental information, specifically non-standard variables, to parent records in the substance use domain. 